\title{
Beam Steering Using Quadrupoles As Position Monitors
}

\author{
Jean-Yves Hémery and Thomas Pettersson \\ CERN \\ CH 1211 - Geneva 23
}

\section{Abstract}

An algorithm is proposed to center the beam in the quadrupoles of a transfer line, by changing the focussing strength of a quadrupole and observing the resulting position shift on a monitor downstream. The observed position shift depends linearly on the beam position offset in the quadrupole, provided the phase advance between the quadrupole and the monitor is not a multiple of $\pi$. The same monitor may thus be used to center the beam in several places of the beam line.

The centering accuracy depends on the resolution of the monitor and on the quadrupole current increment which can be set in such a way that the beam profile remains sufficiently peaked to determine its mean position.

\section{INTRODUCTION}

The tight lay-out of the CERN LEAR experimental area (ref. [1]) is such that an extra means of beam position monitoring was necessary. Quadrupoles are required to transport the beam and can be used as diagnostics when they are associated to one real position monitor. Thus, we inherited a number of extra monitors, which are to be added to the 24 real ones.

A simple software program based on an iterative process is used to center the beam. It implies very simple arithmetic and decision logic criteria to minimize the dipolar effect of a quadrupole on a non-centered beam.

\section{A. Why not another method?}

Solving the problem analytically would have required a larger amount of work. Namely:

- matrix manipulations to solve the system of linear equations.

- data base initialization, 68 magnets +24 monitors

- data base maintenance since the transfer lines lay-out are regularly modified to suit the requests of the experiments.

The authors would like to thank Thys Risselada for helpful discussions and valuable suggestions, and Daniel Jean Simon for his interest and permanent support.
With the method being used, the influence of the following parameters is easy to see:

drift space, magnetic length, focussing power, fringe fisld, alignment error and parasitic field.

The latter is not negligible on a $20 \mathrm{MeV} / \mathrm{c}$ momentum bean ( $1 \mathrm{G}$ over $1 \mathrm{~m}$ gives $1.5 \mathrm{mrad}$ deflection).

The number of measured points required to solve the system of equations would be larger than the necessary number of steps to converge with the present method used.

\section{DESCRIPTION OF THE ALGORITHM}

Let us take (going from upstream) a pair of correctors, a quadrupole and a classical beam position monitor. Only one plane is treated here, but the same logic remains valid for the two of them.

Focussing strength scan sequence:

1: observe beam position on monitor

2: change quadrupole setting

3: observe beam position shift relative to '1:'

4: restore quadrupole nominal setting

How many pieces of information are available from a non-centered beam in a quadrupole?

When scanning, a beam position shift is observed at the monitor located further downstream. It is up to the operator to choose whether a focussing or defocussing is more adequate, depending on the monitor acceptance and resolution, relative to the beam size.

The signs of the $(x, y)$ position shifts tell in which quadrant the beam is passing in the quadrupole.

From this, one can apply a correction using the 2 upstream correctors in the considered plane and re-iterate the loop until the observed shift is zero and the beam centered at the monitor.

The sign and amplitude of the beam shift compared to the ones observed at the previous iteration tell whether the process converges. 
Figure 1 gives a steering in 3 steps. Correctors $a, b$ are tuned according to the beam position on M1 monitor and the shift observed while scanning Q1.

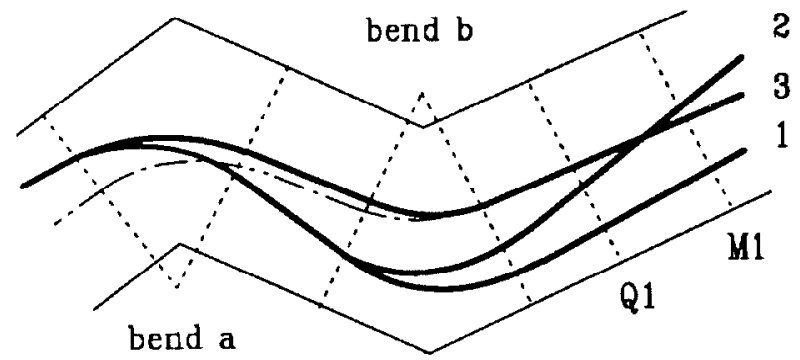

Figure 1: steering in 3 steps

$$
\begin{aligned}
& \text { step } 1: \text { beam position }>0 \text { at monitor, and } \\
& <0 \text { shift when scanning } \\
& \Rightarrow \text { increase bending angle } b
\end{aligned}
$$

\section{DECISION TABLES}

\section{A. Example with 1 quadrupole}

Table 1 supplies the set of decisions to be taken. Fach decision is quoted as a numerical value and corresponds to a change listed below.

The beam position measured at the monitor (while facing downstream the line) before scanning gives one P1 row selected.

Conventions for P1 rows:

$>0 \Rightarrow$ beam in the right hand or upper side

$=0 \Rightarrow$ beam in the center

$<0 \Rightarrow$ beam in the left hand or lower side

The sign of the beam position shift when scanning gives one $\mathrm{S} 1$ column selected.

Conventions for $\mathbf{S 1}$ columns:

$>0 \Rightarrow$ shift to the right or up

$=0 \Rightarrow$ no shift observed

$<0 \Rightarrow$ shift to the left or down warning: the sign of the beam shift has to be inverted in case the quadrupole scan consists in decreasing the focussing or increasing the defocussing strength.

This pair of values supplies the row and column coordinates, and leads to a unique decision.

\begin{tabular}{|c||c|c|c|}
\hline$P 1 \backslash S 1$ & $<0$ & $=0$ & $>0$ \\
\hline \hline$<0$ & 1 & 4 & 4 \\
\hline$=0$ & 3 & 0 & 4 \\
\hline$>0$ & 3 & 3 & 2 \\
\hline
\end{tabular}

Table 1: 1 quadrupole +1 monitor

Meaning of the decision numerical values:

0: last iteration, the beam is centered in the quadrupole and at the monitor

1: $\quad v_{b_{j+1}}=v_{b j}+i_{b} \times d_{b} \times s_{b}$

2: $\quad v_{b_{j+1}}=v_{b_{j}}-i_{b} \times d_{b} \times s_{b}$

3: $\quad v_{b_{j+1}}=v_{b_{j}}-i_{b} \times d_{b} \times s_{b}$

$v_{a_{j+1}}=v_{a_{j}}+i_{a} \times d_{a} \times s_{a}$

4: $\quad v_{b_{j+1}}=v_{b_{j}}+i_{b} \times d_{b} \times s_{b}$

$v_{a_{j+1}}=v_{a,}-i_{a} \times d_{a} \times s_{a}$

\section{With}

$v$ : bending magnet setting value $v_{a}$ and $v_{b}$ are respectively for the upstream and downstream bending magnets $j$ is the iteration index number

$i$ : bending magnet incremenl value

$d: \quad+1$ for deflection to the left or down, -1 for deflection to the right or up

$s$ : respectively the sign of $v_{a}$ or $v_{b_{j}}$

\section{B. Example with 2 quadrupoles}

This case is very similar to the previous one. The meaning of the decision numerical values as well as the conventions remain identical. Only the signs are changed for the $\mathrm{S} 2$ rows of table 2 compared to the $\mathrm{P} 1$ rows in table 1.

\begin{tabular}{|c||c|c|c|}
\hline$S 2 \backslash S 1$ & $<0$ & $=0$ & $>0$ \\
\hline \hline$>0$ & 1 & 4 & 4 \\
\hline$=0$ & 3 & 0 & 4 \\
\hline$<0$ & 3 & 3 & 2 \\
\hline
\end{tabular}

Table 2: 2 quadrupoles +1 monitor 


\section{COARSE AND FINE STEPS}

\section{A. Justification and criteria}

$\odot$ The process does not converge and the results are oscillating (fig.2) with poor damping around the solution and justifies to diminish the step size increments to speed up the convergence.

criterion 1: the two entries in table 1 corresponding to the states 1 and 2 are nol neighbours.

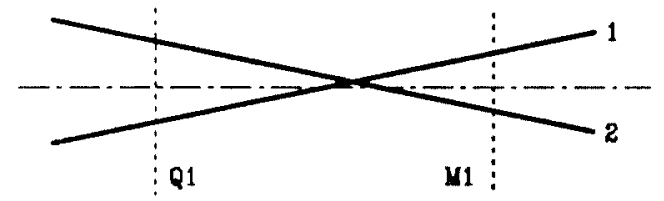

Figure 2: see-saw convergence

C. The program may converge so slowly (fig.3) that it is worth expanding the step size increment on the correctors to specd up the convergence.

criterion 2: the two entries in table 1 corresponding to the states 1 and 2 are the same, and converging.

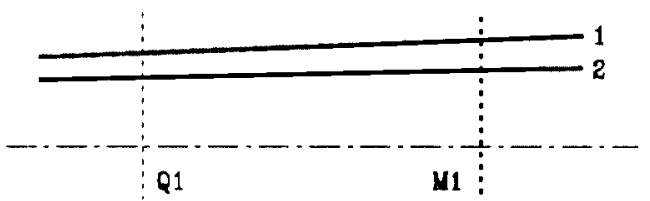

Figure 3: turtle convergence

6 The program may diverge if the transition is from state 2 to 1 (fig. 3 ). The increment $i_{a, b}$ are to be negated.

criterion 3: the two entries in table 1 corresponding to the states 2 and 1 are the same, but diverging.

\section{B. Neighbouring check Implementation}

Each entry of table 3 contains its (row,column) coordinates:

\begin{tabular}{|c|c|c|}
\hline 1,1 & 1,2 & 1,3 \\
\hline 2,1 & $\ldots$ & 2,3 \\
\hline 3,1 & 3,2 & 3,3 \\
\hline
\end{tabular}

Table 3: $(m, n)$ coordinates used for 'neighbouring check'

By evaluating:

$$
N=\left|m_{j}-m_{j-1}\right|+\left|n_{j}-n_{j-1}\right|
$$

in which $j$ is the iteration index number.
The step size increments are altered, depending on the value of $N$ :

\begin{tabular}{|c|c|c|}
\hline$N$ & action & comment \\
\hline$=0$ & $i_{a, b}=i_{a, b} \times 1.1$ & speed up \\
\hline$=1$ & $i_{a, b}=i_{a, b}$ & keep going \\
\hline$>1$ & $i_{a, b}=i_{a, b} \times 0.9$ & slow down \\
\hline
\end{tabular}

Table 4: criteria for step size increments alteration

\section{NO POSITION SHIFT WHILE SCANNING}

The reasons of an undetectable position shift on the monitor while scanning may come from the resolution of the monitor, the weak scan amplitude or limited phase advance close to a multiple of $\pi$ between the quadrupole and the monitor.

The above statement is simply deduced from the wellknown expression:

$$
\Delta x_{M}=x_{\odot} \Delta K l \sqrt{\beta_{Q} \beta_{M}} \sin \left(\mu_{\odot}-\mu_{M}\right)
$$

Where

$$
\begin{array}{ll}
x_{Q} & : \text { beam position at the quadrupole } \\
\Delta K & : \text { quadrupole strength increment } \\
l & : \text { quadrupole length } \\
\beta, \mu & : \text { lattice function values at } Q \text { and } M \text { positions }
\end{array}
$$

About $60 \%$ of the 28 quadrupoles in the LEAR experimental area have betatron phases which allow them to produce a measurable displacement on the associated monitor without provoking beam loss on the vacuum chamber.

\section{RESUlts}

The procedure is successfully applied to center the beam position along the LEAR transfer lines, and on the target of the experiments with zero angle. An accuracy of $0.1 \mathrm{~mm}$ and $20 \mu$ radian on a target is obtained in 6 iterations per plane.

\section{REFERENCES}

[1] D. Dumollard, J-Y. Hémery and D.J. Simon, The LEAR Experimental Areas, Present Lay-out. CERN/PS/91-13(PA). 